\title{
A prospective cohort study of the psychological consequences of the COVID-19 pandemic on frontline healthcare workers in New York City
}

\author{
Lauren A. Peccoralo ${ }^{1,2}$ (D) Robert H. Pietrzak ${ }^{4,5,6}$. Jordyn H. Feingold ${ }^{3}$. Shumayl Syed ${ }^{2} \cdot$ Chi C. Chan $^{3,7}$. \\ James W. Murrough ${ }^{3,8,9} \cdot$ Carly Kaplann $^{2}$. Jaclyn Verity ${ }^{1} \cdot$ Adriana Feder $^{3} \cdot$ Dennis S. Charney ${ }^{3,9}$. \\ Steven M. Southwick ${ }^{5}$. Jonathan A. Ripp ${ }^{1,2}$
}

Received: 23 September 2021 / Accepted: 6 January 2022 / Published online: 22 January 2022

๑) The Author(s), under exclusive licence to Springer-Verlag GmbH Germany, part of Springer Nature 2022

\begin{abstract}
Objectives We sought to describe the course and correlates of psychological distress in frontline healthcare workers (FHCWs) during the COVID-19 pandemic in New York City (NYC).

Methods A prospective cohort study of FHCWs at the Mount Sinai Hospital was conducted during the initial 2020 surge (T1) and 7 months later (T2). Psychological distress [i.e., positive screen for pandemic-related post-traumatic stress disorder (PTSD), major depressive disorder (MDD), and/or generalized anxiety disorder (GAD)], occupational and personal exposures to COVID-19, coping strategies, and psychosocial characteristics were assessed. Four courses of psychological distress response were identified: no/minimal, remitted, persistent, and new-onset. Multinomial logistic regression and relative importance analyses were conducted to identify factors associated with courses of distress.

Results Of 786 FHCWs, 126 (16.0\%) FHCWs had persistent distress; 150 (19.1\%) remitted distress; 35 (4.5\%) new-onset distress; and 475 (60.4\%) no/minimal distress. Relative to FHCWs with no/minimal distress, those with persistent distress reported greater relationship worries [19.8\% relative variance explained (RVE)], pre-pandemic burnout (18.7\% RVE), lower dispositional optimism (9.8\% RVE), less emotional support (8.6\% RVE), and feeling less valued by hospital leadership (8.4\% RVE). Relative to FHCWs with remitted symptoms, those with persistent distress reported less emotional support (29.7\% RVE), fewer years in practice (28.3\% RVE), and psychiatric history (23.6\% RVE).

Conclusions One-fifth of FHCWs in our study experienced psychological distress 7 months following the COVID-19 surge in NYC. Pandemic-related worries, pre-pandemic burnout, emotional support, and feeling valued by leaders were linked to persistent distress. Implications for prevention, treatment, and organizational efforts to mitigate distress in FHCWs are discussed.
\end{abstract}

Keywords Psychological distress · COVID-19 pandemic $\cdot$ Healthcare workers $\cdot$ Mental Health $\cdot$ Burnout

Lauren A. Peccoralo

lauren.peccoralo@mountsinai.org

1 Department of Medicine, Icahn School of Medicine at Mount Sinai, One Gustave L. Levy Place, Box 1087, New York, NY 10029, USA

2 Department of Medical Education, Icahn School of Medicine at Mount Sinai, New York, NY, USA

3 Department of Psychiatry, Icahn School of Medicine at Mount Sinai, New York, NY, USA

4 U.S. Department of Veterans Affairs National Center for Posttraumatic Stress Disorder, VA Connecticut Healthcare System, West Haven, CT, USA
5 Department of Psychiatry, Yale School of Medicine, New Haven, CT, USA

6 Department of Social and Behavioral Sciences, Yale School of Public Health, New Haven, CT, USA

7 Mental Illness Research, Education, and Clinical Center, James J. Peters VA Medical Center, Bronx, NY, USA

8 Depression and Anxiety Center for Discovery and Treatment, New York, USA

9 Department of Neuroscience, Icahn School of Medicine at Mount Sinai, New York, USA 


\section{Introduction}

The COVID-19 pandemic has placed a significant strain on frontline healthcare workers (FHCWs), especially those who cared for COVID patients during the initial pandemic surges (Dong et al. 2020). While FHCWs exhibit considerable resilience (Alahdab et al. 2020; Cai et al. 2020; Peccoralo et al. 2021; Tabakakis et al. 2019), they may also be particularly vulnerable to adverse mental health effects of the pandemic, given the intensity and prolonged nature of their occupational exposures. Psychological consequences of disasters on medical responders commonly include depression and posttraumatic stress disorder (PTSD) (Naushad et al. 2019), and in some instances, symptoms may persist for years beyond the event (Wu et al. 2009).

During the COVID-19 pandemic, prevalence of psychological distress, including generalized anxiety disorder (GAD) and major depressive disorder (MDD), in FHCWs working in NYC during the surge, was alarmingly high (Feingold et al. 2021; Schwartz et al. 2021; Shechter et al. 2020; Forrest et al. 2021). We previously reported that nearly $40 \%$ of FHCWs experienced significant MDD, GAD, and/or PTSD symptoms (Feingold et al. 2021), while others found that 1 in 5 resident physicians contemplated suicide or self-harm in the wake of the initial COVID-19 surge (Schwartz et al. 2021). A cross-sectional study of 14,600 US healthcare workers found that $41 \%$ felt burned out, $38 \%$ experienced anxiety, and $21 \%$ reported sadness (Forrest et al. 2021), while FHCWs in Wuhan, China, experienced high rates of distress $(71.5 \%)$, depression (50.4\%), and anxiety (44.6\%; Lai et al. 2020).

Studies of the general population and HCWs following various pandemics have found that certain mental health symptoms may persist months and even years after the acute stage of the crisis (O'Connor et al. 2020; Wang et al. 2020; Sasaki et al. 2021; Feder et al. 2016; Paladino et al. 2017). Three longitudinal studies of the general population during COVID-19, from China, Belgium, and the United Kingdom, demonstrated relatively stable levels of anxiety and depression between time points, although these studies spanned a total survey time of 8 weeks or less (O'Connor et al. 2020; Wang et al. 2020). A study on the impact of COVID-19 on HCWs in Japan showed sustained levels of elevated psychological distress across an eight-month period (Sasaki et al. 2021).

Investigations of the psychological impact on FHCWs working during novel viral outbreaks, including COVID19, have demonstrated a number of factors associated with psychological distress, including greater exposure to infected patients, perceived lack of organizational support, time spent in quarantine, and younger age (Bohlken et al. 2020; Kisely et al. 2020). In addition, studies of the mental health impact on medical first responders across multiple disasters showed that limited social support, use of maladaptive coping strategies, and lack of training were associated with developing psychological distress (Bohlken et al. 2020; Cai et al. 2020).

While these studies inform our understanding of the burden of mental health problems on both the general population and FHCWs during and immediately following the COVID-19 pandemic surge, no known study to date has examined longitudinal trends in the mental health impact of COVID-19 on FHCWs in the US (O'Connor et al. 2020; Wang et al. 2020). In addition, few studies have assessed symptoms months later and/or the myriad of factors that may impact the persistence or remittance of psychological distress in this group.

To address this gap, we sought to describe the predominant courses of psychological distress among FHCWs during and following the COVID-19 pandemic surge in NYC, and to identify demographic, professional, coping and behavioral factors, and COVID-19-related personal and work-related factors associated with these courses over time. Our previous work on the psychological impact of COVID-19 on FHCWs during the acute surge revealed that pre-pandemic burnout, history of mental illness, being high medical risk, experiencing moral distress, and lower perceived support from leadership were all associated with an increased likelihood of having psychological distress (Feingold et al. 2021). The present study is a prospective follow-up of that cohort 7 months later to examine the trajectory of their psychological distress and the associated factors. A systematic review of resilience and psychological dysfunction after trauma found that among those exposed to potentially traumatic events, $65.7 \%$ never developed psychological symptoms, $20.8 \%$ recovered from symptoms, $10.9 \%$ had chronic symptoms, and $8.9 \%$ had delayed onset of mental health symptoms (Galatzer-Levy et al. 2018). Those who never develop symptoms and those who recover from symptoms might be deemed more resilient than the persistent and new-onset groups; however, symptom course may also be related to intensity of exposures. We therefore use terms without reference to resilience in our report: no/minimal (never), remitted (recovered), persistent (chronic), and new-onset (delayed) distress. We hypothesized similar prevalence of symptom courses within the 7-month follow-up period, and that certain factors, such as greater medical risk, psychiatric history, pre-pandemic burnout, maladaptive coping strategies, lower perceived social and leadership support, and greater COVID-19-related exposures, would be associated with persistent and new-onset psychological distress in FHCWs. We evaluated 2 research questions:

Research question 1: what is the prevalence of longitudinal courses of mental health (GAD, MDD, PTSD) distress (no/minimal, persistent, remitted, new-onset)? 
Research question 2: what are the demographic, occupational, COVID-19 pandemic related, and psychosocial characteristics associated with persistent (vs. no/minimal and remitted distress) and new-onset distress (vs. no/minimal distress)?

\section{Methods}

\section{Participants and Timeline}

In this prospective cohort study, FHCWs at the Mount Sinai Hospital (MSH), an urban tertiary care hospital in NYC, were asked to participate in two surveys: one during the initial pandemic peak from April 14, 2020 to May 11, 2020 [time 1 (T1)] and another at 7-month follow-up [time 2 (T2)] from November 19, 2020 to January 11, 2021. Our initial aim was to conduct a survey 6 months post-surge to assess the long-term impact of the initial surge on our FHCWs; however, the $\mathrm{T} 2$ survey distribution was delayed briefly due to modifications to the IRB proposal. Both surveys were constructed on the Research Electronic Data Capture (REDCap) platform and administered anonymously, with links sent via email to eligible participants. Comprehensive methods for the T1 survey are detailed elsewhere (Feingold et al. 2021). T1 corresponded with the initial peak and downward slope of the pandemic curve at MSH, according to COVID19 inpatient census data. T2 corresponded to a secondary rise and plateau of the pandemic curve at MSH. Despite this rise in cases, there were far fewer redeployments during $\mathrm{T} 2$ (2.9\% compared to $36.4 \%$ during $\mathrm{T} 1$ ) and the hospital system was largely functioning as it had pre-pandemic with ambulatory services open and elective surgeries resumed. In addition, to distinguish any new stressors that might influence persistent or new symptoms, we added additional questions to address current COVID-19- and work-related factors and their impact on symptoms (Supplemental Table 1).

We attempted to contact the entire $\mathrm{T} 1$ sample via email at T2 for follow-up assessment. For each survey, participants self-generated a six-digit alphanumeric research code from the same five prompts eliciting letters or digits from unchanging personal historical facts (e.g., first letter of mother's maiden name). This was designed to enable an approximate deterministic data linkage method to link T1 and $\mathrm{T} 2$ surveys while preserving anonymity (Dusetzina et al. 2014). A T2 survey was matched to a T1 survey if (1) the self-generated research codes were an exact match $(n=574)$ or (2) the self-generated research codes were within one generalized Levenshtein edit distance (the minimal possibly weighted number of insertions, deletions, or substitutions need to transform one string into another; evaluated using the "agrep" function in R) (Levenshtein 1966) and the surveys matched on at least 4 out of 5 of the following demographic variables: age category, gender, profession, department, and years in practice.

The eligible study population for both surveys included HCWs directly involved in the care of patients infected with COVID-19 at T1 as a result of their standard scope of practice or redeployment assignment within the study period (Feingold et al. 2021). The sample included attending-level physicians, residents, and clinical fellows from several departments, patient-facing nurses and nurse practitioners, physician assistants, chaplains, clinical psychologists, social workers, and dietitians. Participants were excluded if email invitations went undelivered to addresses on file (no longer working at MSH). At T2, eligible participants were given the opportunity to receive electronic products via raffle by completing a separate unlinked form following completion of the $\mathrm{T} 2$ survey. This study was approved by the Institutional Review Board at the Icahn School of Medicine at Mount Sinai.

\section{Study instruments}

Survey items assessed at T1 and T2 are described in Supplemental Table 1. The T1 survey assessed demographics, professional characteristics, COVID-19-associated personal and occupational exposures, coping and restorative behaviors, and psychosocial characteristics, pre-pandemic (West et al. 2009, 2012), and current psychological symptoms. The T2 survey included items assessing post-acute personal, occupational, and COVID-19-related exposures, other psychological distress symptoms, as well as race/ethnicity (not assessed in T1).

\section{Outcome measures}

Psychological Distress. Psychological distress was defined as screening positive on at least one of the three psychological symptom measures administered on our surveys, including COVID-19-related posttraumatic stress disorder (C19-PTSD), major depressive disorder (MDD), and generalized anxiety disorder (GAD). Positive screens on these disorders were combined, as scores on the prevalence of positive screens overlapped substantially, scores on scales used to assess them loaded strongly on a single factor, and because doing so increased statistical power in analyses of less prevalent symptom courses (e.g., new-onset distress).

Symptoms of C19-PTSD were assessed using the 4-item PTSD Checklist for DSM-5 (PCL4-5) (Geier et al. 2020) at T1 and the full, 20-item PTSD Checklist for DSM-5 (PCL-5) at T2 (Weathers et al. 2013) to obtain a more comprehensive assessment of PTSD symptoms, and both were modified to assess COVID-19 pandemic-related exposure. A score of $\geq 8$ on the PCL4-5 at T1 was considered a positive screen (Geier et al. 2020); a score $\geq 33$ on the PCL-5 at T2 was considered 
a positive screen (Weathers et al. 2013); and both were calibrated against the gold-standard CAPS- 5 assessment of PTSD diagnosis (Geier et al. 2020). In the current sample, internal consistency of PCL items was good to excellent $(\mathrm{T} 1 \alpha=0.82, \mathrm{~T} 2 \alpha=0.95)$.

MDD symptoms were assessed using the 8-item Patient Health Questionnaire-8 (PHQ-8) (Shin et al. 2019). A score $\geq 10$ on the PHQ- 8 was considered a positive screen, which correctly classified $96.5 \%$ of individuals with MDD and related disorders in a validation study (Shin et al. 2019). In the current sample, internal consistency of PHQ-8 items was good to excellent ( $\mathrm{T} 1 \alpha=0.86, \mathrm{~T} 2 \alpha=0.90)$.

GAD symptoms were assessed using the 7-item Generalized Anxiety Disorder-7 (GAD-7) questionnaire (Spitzer et al. 2006). A score $\geq 10$ was considered a positive screen, which has a sensitivity of 0.89 and specificity of 0.82 in diagnosing GAD (Spitzer et al. 2006). In the current sample, internal consistency of GAD-7 items was excellent (T1 $\alpha=0.91, \mathrm{~T} 2 \alpha=0.91)$.

\section{Data analysis}

To evaluate the representativeness of our sample, we compared demographic and occupational characteristics of respondents in $\mathrm{T} 2$ to both non-completers of the T2 survey from the T1 survey, as well as to our institution's human resources database of $\mathrm{HCWs}$ from major occupational groups in the study sample (e.g., residents/fellows, RNs, attending physicians) using Chi-square analyses.

To evaluate Research Question 1, we computed the prevalence of positive screens for psychological distress at both time points. Four groups were then created based on presence/absence of mental health symptoms at $\mathrm{T} 1$ and/or T2: No/minimal distress: negative screens for distress $\mathrm{T} 1$ and $\mathrm{T} 2$; Remitted distress: positive screen for distress at $\mathrm{T} 1$ but not T2; Persistent distress: positive screen for distress at $\mathrm{T} 1$ and T2; and New-onset distress: negative screen for distress at $\mathrm{T} 1$ and positive screen for distress at T2. Exploratory factor analyses were conducted to generate factor scores $(0=$ mean, $1=\mathrm{SD}$ ) of measures of acute (T1) and post-acute (T2) COVID-related stressors as well as acute (T1) infection-, family-, and work-related concerns, protective psychosocial characteristics, work-related inspiration, and feeling valued/ supported at work (Supplemental Table 1).

To evaluate Research Question 2, we began with a Chisquare analyses and analyses of variance with Bonferronicorrected pairwise contrasts to determine bivariate-level group differences in sample characteristics. We then conducted a multinomial logistic regression analysis with backward elimination to identify factors associated with persistent and new-onset distress relative to no/low distress and remitted distress variables (including only those factors associated with distress group in bivariate analyses at the $p<0.05$ level). To identify T1 variables associated with symptomatic distress at T2, analyses focused on predictors of persistent and new-onset distress. Planned post hoc analyses of multi-indicator variables (e.g., COVID-19 stressors) were conducted to identify component variables that were associated with persistent and new-onset distress. Finally, we conducted relative importance analyses (Tonidandel and LeBreton 2009) to identify the relative proportion of variance in persistent and new-onset distress that was explained by each of the significant independent variables identified in the multinomial analyses. Missing data were multiply imputed prior to analysis using chained equations (Azur et al. 2011). All analyses were conducted using IBM SPSS Statistics for Windows, Version 27.0. IBM Corp. Released 2020 (Armonk, NY); and the R statistical software package relaimpo was used to conduct the relative importance analysis (Groemping 2006).

\section{Results}

Of the 6,026 recruited FHCWs, 3,360 completed the T1 survey $(55.8 \%)$, of which 2,579 (76.8\%) endorsed frontline responsibilities; median completion date $=04 / 21 / 20$; and range $=04 / 14 / 20-05 / 11 / 20$ (T1 sample characteristics detailed in Feingold et al. 2021). A total of 786 (30.5\%) FHCWs who completed the T1 assessment completed the T2 follow-up assessment (median completion date $=12 / 6 / 20 ;$ range $=11 / 19 / 20-1 / 10 / 21)$. Distributions of age, sex, profession, marital and parental status, leadership and redeployment status, and pre-pandemic psychiatric history between $\mathrm{T} 2$ completers and non-completers did not differ (all $\chi^{2}<1.32$, all $p$ 's $>0.20$ ). Comparison of gender and race/ethnicity distributions in major occupational groups in the study sample (e.g., residents/fellows, RNs, attending physicians) did not differ relative to those observed in Mount Sinai Hospital human resources data (all $p$ 's $>0.10$ ).

\section{Research question 1: prevalence of longitudinal courses of psychological distress}

Of the 786 who completed both T1 and T2 surveys, a total of $475(60.4 \%)$ FHCWs did not screen positive for distress at either time point; $150(19.1 \%)$ had remitted distress; 126 (16.0\%) had persistent distress; and 35 (4.5\%) had new-onset distress. Table 1 shows characteristics of the full sample and categorization according to the different courses of distress. The distress courses differed significantly with respect to all of the variables assessed, with the exception of race/ethnicity and work-related inspiration. 
Table 1 Characteristics of the sample and courses of psychological distress in healthcare workers on the frontlines of the COVID-19 pandemic

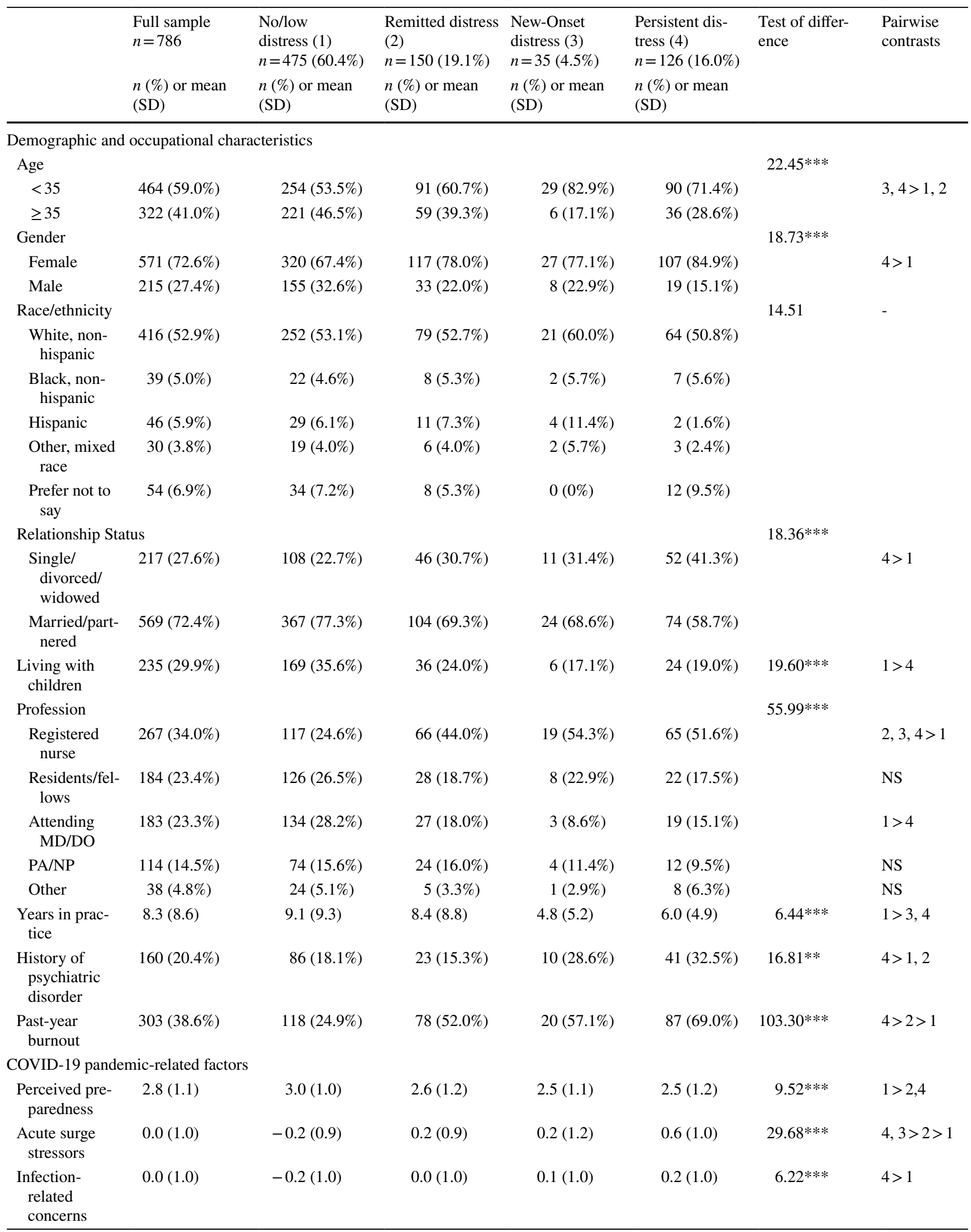


Table 1 (continued)

\begin{tabular}{|c|c|c|c|c|c|c|c|}
\hline & $\begin{array}{l}\text { Full sample } \\
n=786\end{array}$ & $\begin{array}{l}\text { No/low } \\
\text { distress (1) } \\
n=475(60.4 \%)\end{array}$ & $\begin{array}{l}\text { Remitted distress } \\
(2) \\
n=150(19.1 \%)\end{array}$ & $\begin{array}{l}\text { New-Onset } \\
\text { distress (3) } \\
n=35(4.5 \%)\end{array}$ & $\begin{array}{l}\text { Persistent dis- } \\
\text { tress (4) } \\
n=126(16.0 \%)\end{array}$ & $\begin{array}{l}\text { Test of differ- } \\
\text { ence }\end{array}$ & $\begin{array}{l}\text { Pairwise } \\
\text { contrasts }\end{array}$ \\
\hline & $\begin{array}{l}n(\%) \text { or mean } \\
(\mathrm{SD})\end{array}$ & $\begin{array}{l}n(\%) \text { or mean } \\
\text { (SD) }\end{array}$ & $\begin{array}{l}n(\%) \text { or mean } \\
\text { (SD) }\end{array}$ & $\begin{array}{l}n(\%) \text { or mean } \\
\text { (SD) }\end{array}$ & $\begin{array}{l}n(\%) \text { or mean } \\
(\mathrm{SD})\end{array}$ & & \\
\hline $\begin{array}{l}\text { Family-related } \\
\text { concerns }\end{array}$ & $0.0(1.0)$ & $-0.3(1.0)$ & $0.3(1.0)$ & $-0.1(1.2)$ & $0.2(1.1)$ & $16.94 * * *$ & $2,4>1$ \\
\hline $\begin{array}{l}\text { Work-related } \\
\text { concerns }\end{array}$ & $0.0(1.0)$ & $-0.2(0.9)$ & $0.3(1.0)$ & $0.0(1.0)$ & $0.4(1.0)$ & $20.01 * * *$ & $2,4>1$ \\
\hline $\begin{array}{l}\text { Post-acute } \\
\text { surge stress- } \\
\text { ors }\end{array}$ & $0.0(1.0)$ & $-0.2(0.9)$ & $-0.1(0.8)$ & $0.8(1.1)$ & $0.6(1.2)$ & $28.12 * * *$ & $4,3>1,2$ \\
\hline \multicolumn{8}{|c|}{ Psychosocial characteristics } \\
\hline $\begin{array}{l}\text { Coping self- } \\
\text { efficacy } \\
\text { (resilience) }\end{array}$ & $6.5(1.3)$ & $6.7(1.3)$ & $6.2(1.3)$ & $6.6(1.3)$ & $6.1(1.3)$ & $12.40 * * *$ & $1>2,4$ \\
\hline $\begin{array}{l}\text { Positive charac- } \\
\text { teristics }\end{array}$ & $0(1.0)$ & $0.2(0.9)$ & $-0.1(1.0)$ & $-0.2(1.1)$ & $-0.4(1.1)$ & $13.77 * * *$ & $1>2,4$ \\
\hline $\begin{array}{c}\text { Work-related } \\
\text { inspiration }\end{array}$ & $0(1.0)$ & $0.1(1.0)$ & $-0.1(1.0)$ & $-0.1(1.0)$ & $-0.1(1.1)$ & 1.63 & - \\
\hline $\begin{array}{l}\text { Feeling valued/ } \\
\text { supported at } \\
\text { work }\end{array}$ & $0(1.0)$ & $0.2(0.9)$ & $-0.3(1.0)$ & $-0.4(1.0)$ & $-0.5(1.0)$ & $26.72 * * *$ & $1>2,3,4$ \\
\hline $\begin{array}{l}\text { Perceived } \\
\text { social support }\end{array}$ & $12.3(2.9)$ & $12.8(2.6)$ & $12.2(2.7)$ & $11.4(3.5)$ & $11.1(3.3)$ & $13.37 * * *$ & $1>3,4$ \\
\hline \multicolumn{8}{|c|}{ Restorative behaviors } \\
\hline Sleep hours & $6.4(1.1)$ & $6.6(1.0)$ & $6.3(1.3)$ & $6.6(1.1)$ & $6.0(1.1)$ & $10.79 * * *$ & $1>2,4$ \\
\hline $\begin{array}{l}\text { Physical exer- } \\
\text { cise }\end{array}$ & $2.2(1.9)$ & $2.4(2.0)$ & $2.0(1.8)$ & $2.2(1.9)$ & $1.9(1.8)$ & $2.69 *$ & NS \\
\hline \multicolumn{8}{|l|}{ Coping strategies } \\
\hline $\begin{array}{l}\text { Self-sufficient } \\
\text { coping }\end{array}$ & $1.4(1.1)$ & $1.6(1.0)$ & $1.1(1.0)$ & $1.2(1.3)$ & $1.1(1.0)$ & $12.33 * * *$ & $1>2,4$ \\
\hline $\begin{array}{l}\text { Socially sup- } \\
\text { ported coping }\end{array}$ & $1.0(0.7)$ & $0.9(0.7)$ & $1.2(0.7)$ & $0.9(0.7)$ & $1.0(0.7)$ & $6.62 * * *$ & $2>1$ \\
\hline $\begin{array}{l}\text { Avoidant cop- } \\
\text { ing }\end{array}$ & $0.8(0.6)$ & $0.7(0.6)$ & $0.8(0.6)$ & $1.0(0.7)$ & $1.0(0.7)$ & $9.48 * * *$ & $4>1$ \\
\hline
\end{tabular}

Distress $=$ psychological distress (positive screen for MDD, GAD, and PTSD symptoms)

Statistically significant association: $* p<0.05, * * p<0.01, * * * p<0.001$

\section{Research question 2: factors associated with symptomatic persistent and new-onset distress}

\section{Persistent distress vs. no/minimal distress}

Table 2 shows results of multinomial logistic regression analyses of factors associated with persistent distress. Relative to the no/minimal distress group, the persistent distress group was more likely to be female, has a history of a psychiatric disorder, and reports pre-pandemic burnout; they also reported greater severity of post-acute stressors and infection-related, family-related, and work-related concerns during T1. The persistent distress group scored lower on measures of protective psychosocial characteristics, reported feeling less valued/supported at work and less social support, while being also being more likely to utilize avoidant coping strategies. Our planned post hoc analyses revealed that several acute and post-acute variables, including worries, coping behaviors, feelings of value and support, having to make difficult decisions, and personal medical risk were associated with more persistent distress when compared with the no/ low symptoms group (Table 3).

A relative importance analysis indicated that greater worries about the effect of the pandemic on personal relationships [19.8\% relative variance explained (RVE)], prepandemic burnout (18.7\% RVE), and lower dispositional optimism (9.8\% RVE), emotional support (8.6\% RVE), and feelings of being valued by hospital leadership (8.4\% RVE) during the acute surge, collectively explained the majority 
Table 2 Results of multinomial logistic regression model predicting a persistent course of psychological distress in healthcare workers on the frontlines of the COVID-19 pandemic

\begin{tabular}{lll}
\hline & $\begin{array}{l}\text { Persistent distress vs no/low } \\
\text { distress } \\
R^{2}=0.55 \\
\text { RRR }(95 \% \mathrm{CI})\end{array}$ & $\begin{array}{l}\text { Persistent distress vs } \\
\text { remitted distress } \\
R^{2}=0.31\end{array}$ \\
& & RRR $(95 \% \mathrm{CI})$ \\
\hline Demographic and occupational characteristics & $2.30(1.17-4.53)^{*}$ & $1.93(0.92-4.05)$ \\
Female gender & $0.56(0.27-1.15)$ & $1.48(0.69-3.21)$ \\
Have children & $1.73(0.98-3.06)$ & $1.11(0.61-2.00)$ \\
RN vs. other profession & $2.36(1.29-4.30)^{* *}$ & $2.85(1.46-5.56)^{* *}$ \\
History of psychiatric disorder & $3.95(2.36-6.60)^{* * *}$ & $1.62(0.93-2.79)$ \\
Past-year burnout & $0.97(0.93-1.02)$ & $0.95(0.91-0.99)^{*}$ \\
Years in practice & & \\
COVID-19-related variables & $1.43(0.90-2.27)$ & $0.89(0.55-1.46)$ \\
Acute surge stressors & $1.39(1.04-1.86)^{*}$ & $1.79(1.30-2.46)^{* * *}$ \\
Post-acute surge stressors & $1.35(1.03-1.78)^{*}$ & $1.03(0.77-1.38)$ \\
Infection-related concerns & $1.54(1.13-2.11)^{* *}$ & $0.70(0.50-0.98)^{*}$ \\
Family-related concerns & $1.71(1.27-2.29)^{* * *}$ & $0.97(0.71-1.31)$ \\
Work-related concerns & & \\
Psychosocial characteristics & $0.72(0.55-0.94)^{*}$ & $0.97(0.73-1.29)$ \\
Positive dispositional characteristics & $0.71(0.54-0.93)^{*}$ & $0.86(0.65-1.15)$ \\
Feeling valued/supported at work & $0.89(0.81-0.97)^{* *}$ & $0.90(0.82-0.99)^{*}$ \\
Perceived social support & & \\
Restorative behaviors & $0.81(0.64-1.04)$ & $0.85(0.66-1.09)$ \\
Sleep hours & & $0.73(0.48-1.10)$ \\
Coping strategies & $1.31(0.89-1.93)$ & $1.26(0.81-1.97)$ \\
Socially supported coping & & \\
Avoidant coping & & \\
\hline
\end{tabular}

Distress = psychological distress (positive screen for MDD, GAD, and PTSD symptoms)

$R R R$ Relative Risk Reduction, $R N$ Registered Nurse

Statistically significant association: $* p<0.05, * * p<0.01$, ***p $p<0.001$ of variance in the persistent distress group compared to the no/minimal distress trajectory group (Fig. 1).

\section{Persistent distress vs. remitted distress}

Relative to the remitted distress group, the persistent distress group was more likely to report having a history of a psychiatric disorder, fewer years in practice, greater severity of postacute stressors, more family-related concerns during the acute phase, and less social support during the acute surge. Planned post hoc analyses revealed that lower perception of emotional support and being at a higher medical risk were associated with persistent distress vs remitted distress (Table 3).

A relative importance analysis (Fig. 2) indicated that lower emotional support during the acute surge $(29.7 \%$ RVE), fewer years of practice (28.3\% RVE), and history of mental disorder prior to the pandemic (23.6\% RVE) explained the majority of variance in a persistent vs. remitted distress course (Fig. 2).

\section{New-onset distress vs. no/minimal distress}

Significant predictors of new distress compared to no/ low distress included $\mathrm{RN}$ professional status $(\mathrm{RRR}=3.12$, $95 \% \mathrm{CI}=1.29-7.55)$, fewer years in practice $(\mathrm{RRR}=0.90$, 95\% CI $=0.82-0.99$ ), positive screen for pre-pandemic burnout $(\mathrm{RRR}=2.67,95 \% \mathrm{CI}=1.21-5.86)$, and greater severity of post-acute surge stressors ( $R R R=2.21,95 \%$ $\mathrm{CI}=1.47-3.31$ ). Post hoc analyses revealed that caring for patients who died from the virus at $\mathrm{T} 1$ and personal medical risk at $\mathrm{T} 2$ were associated with new-onset distress (Table 3).

A relative importance analysis indicated that having cared for patients who died (45.8\% RVE), fewer years in practice (23.7\%), pre-pandemic burnout (13.7\% RVE), female gender (12.1\% RVE), and higher personal medical risk (4.7\%) explained the majority of variance in new distress vs. no/ minimal distress course. 
Table 3 Post hoc analysis of acute and post-acute surge variables

\begin{tabular}{|c|c|}
\hline Persistent distress vs no/low distress & $\begin{array}{l}\text { RRR } \\
(95 \% \mathrm{CI})\end{array}$ \\
\hline \multicolumn{2}{|l|}{ Acute surge variables } \\
\hline \multicolumn{2}{|l|}{ Worries } \\
\hline Worries about infecting colleagues & $1.23(1.01-1.53)$ \\
\hline Worries about the effect of the pandemic on personal relationships & $1.66(1.29-2.14)$ \\
\hline Worries about not being able to do enough for COVID-19 patients & $1.51(1.16-1.95)$ \\
\hline \multicolumn{2}{|l|}{ Coping } \\
\hline Substance use & $2.68(1.13-6.3)$ \\
\hline Behavioral disengagement & $3.02(1.08-8.47)$ \\
\hline Dispositional optimism & $0.73(0.61-0.86)$ \\
\hline \multicolumn{2}{|l|}{ Value/support } \\
\hline Felt valued by leadership & $0.72(0.55-0.95)$ \\
\hline Felt emotional support & $0.67(0.53-0.84)$ \\
\hline \multicolumn{2}{|l|}{ Post-Acute Surge Variables } \\
\hline Having to make difficult decisions prioritizing COVID-19 patients & $3.52(1.60-7.75)$ \\
\hline Medically high risk & $4.50(1.53-13.33)$ \\
\hline Persistent distress vs no/low distress & $\begin{array}{l}\text { RRR } \\
(95 \% \text { CI })\end{array}$ \\
\hline \multicolumn{2}{|l|}{ Acute surge variables } \\
\hline Felt emotional support & $0.69(0.54-0.88)$ \\
\hline \multicolumn{2}{|l|}{ Post-acute surge variables } \\
\hline Medically high risk & $4.44(1.42-13.89)$ \\
\hline New distress vs no/low distress & $\begin{array}{l}\text { RRR } \\
(95 \% \text { CI })\end{array}$ \\
\hline \multicolumn{2}{|l|}{ Acute surge variables } \\
\hline Caring for patients who died & $1.71(1.03-3.89)$ \\
\hline \multicolumn{2}{|l|}{ Post-acute surge variables } \\
\hline Medically high risk & $2.52(1.43-6.37)$ \\
\hline
\end{tabular}

Distress = psychological distress (positive screen for MDD, GAD, and PTSD symptoms)

RRR Relative Risk Ratio, 95\% CI 95\% confidence interval

\section{Discussion}

To our knowledge, this study is the first to examine predominant longitudinal courses of psychological distress in FHCWs following exposures related to the COVID-19 pandemic in the US. Our data demonstrate that approximately $60 \%$ never developed depression, anxiety or PTSD symptoms, $19.1 \%$ had remitted symptoms; $16 \%$ had persistent symptoms; and $4.5 \%$ had new-onset symptoms. These findings align with findings from a study in Italy over a one-year time frame with a similar follow-up response rate, showing a range of $40-73 \%$ without symptoms, 16-28\% remitted symptoms, 6-24\% persistent symptoms, and 5-9\% newonset symptoms (Rossi et al. 2021). In contrast, a study of HCWs in Argentina found that mental health symptom rates increased over a 4-month time period, with depression increasing from 40 to $46 \%$ and anxiety from 53 to $63 \%$
(López Steinmetz et al. 2021). Differences across studies may in part be due to the circumstances of the pandemic during the time frames in each country, as well as the shorter time frame for the study in Argentina. Nonetheless, it is clear from all of these studies that there are subsets of FHCWs who have persistent or new-onset psychological distress many months after the initial COVID pandemic surge.

Relative to FHCWs with no/minimal distress, those with persistent distress were four times more likely to report prepandemic burnout and more than twice as likely to have had a pre-pandemic psychiatric disorder, which is consistent with the aforementioned study in Argentina (López Steinmetz et al. 2021). Those who perceived greater support from leadership and greater emotional support during the acute surge were less likely to have persistent distress symptoms relative to the no/minimal distress group. Moreover, FHCWs with fewer years in practice and those with higher personal 


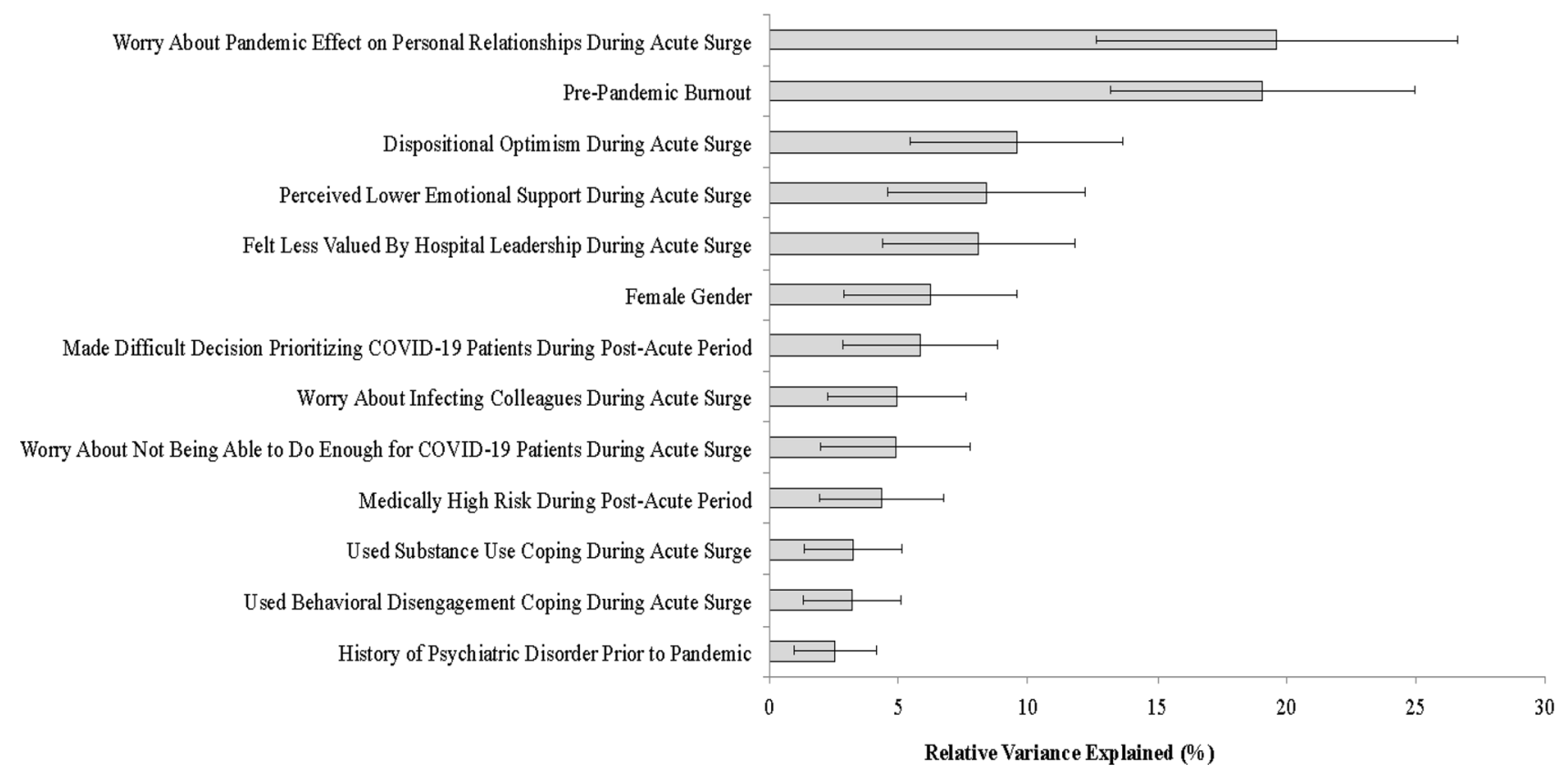

Fig. 1 Results of relative importance analysis of acute and post-acute surge predictors of persistent vs. no/low psychological distress. Note: Error bars represent $95 \%$ confidence intervals

Fig. 2 Results of relative importance analysis of acute and post-acute surge predictors of persistent vs. remitted psychological distress. Note: Error bars represent $95 \%$ confidence intervals; family-related concerns were not significant in this analysis (relative variance explained $<0.5 \%$ )

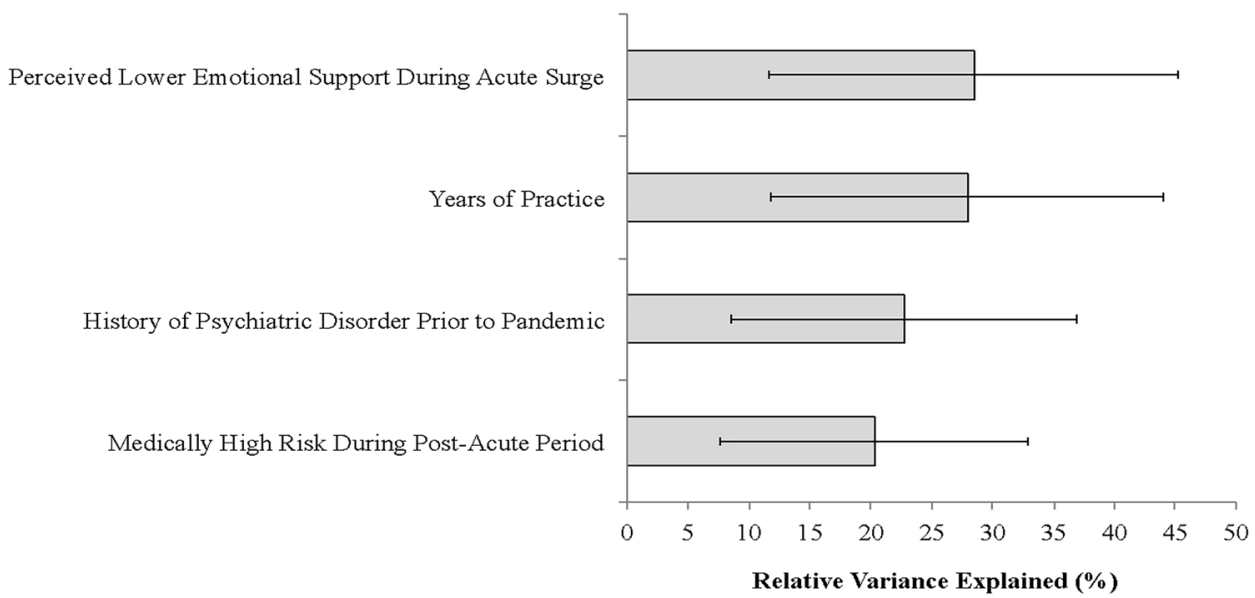

Relative Variance Explained (\%) medical risk at $\mathrm{T} 2$ were more likely to develop persistent or new distress. FHCWs who were less optimistic, disengaged or used substances as a form of coping were more likely to have persistent distress. New-onset of distress was also associated with pre-pandemic burnout, female gender, and most strongly, caring for patients who passed away from COVID-19.

Prior studies, including ours, have found that baseline pre-existing psychiatric disorders, burnout, and stress prior to traumatic events are associated with higher likelihood of developing distress in response to a new stressor (Feder et al. 2016; Feingold et al. 2021; López Steinmetz et al. 2021; Smid et al. 2012, 2013). This finding aligns with studies of disaster responders and survivors, as well as military personnel, which have demonstrated a "stress sensitization" phenomenon in which prior stressors and related distress may result in higher and more persistent post-traumatic distress following exposure to a new traumatic or stressful event (Feder et al. 2016; Smid et al. 2012, 2013). Hence, pre-pandemic psychiatric morbidities and burnout may, in part, "stress sensitize" FHCWs and predispose them to persistent distress following a chronic traumatic event, such as the COVID-19 pandemic. This finding underscores the importance of assessing and promoting initiatives to mitigate psychiatric difficulties and reduce occupational burnout 
on an ongoing basis to prevent distress experienced by the healthcare workforce following communal traumas.

Lower levels of perceived leadership support and general emotional support during the acute pandemic surge were both strongly associated with persistent distress following the COVID-19 surge. This finding is consistent with prior work showing a link between leadership support and wellbeing. In a sample of 2800 physicians, every 1-point increase in composite ratings of leader effectiveness was associated with a 3.3\% reduced odds of burnout (Shanafelt et al. 2015). Further, in a study of $1092 \mathrm{HCWs}$, greater perceived need for social support was associated with higher likelihood of depression, anxiety, and PTSD symptoms (Hennein et al. 2021). This finding also aligns with prior meta-analytic findings suggesting that low level of post-trauma social support is one of the strongest risk factors for PTSD (Ozer et al. 2003). Despite these findings, strong evidence for interventions on how to improve leadership and peer support during a pandemic is sparse. A recent Cochrane review demonstrated moderate confidence that effective, safe, supportive formal and informal communication within organizations may help facilitate successful strategies to reduce FHCW stress during pandemics (Pollock et al. 2020). Institutions may therefore consider developing resources that support leaders and provide them with structures and tools for effective, supportive communication, such as encouraging supervisors to create forums using the "hear me, protect me, prepare me, support me, and care for me" framework (Shanafelt et al. 2020).

Having cared for patients who died from COVID-19 during the acute phase explained the majority of variance in the $4.5 \%$ of FHCWs who developed new-onset distress. In addition, greater worry and guilt during the acute surge were linked to persistent distress. These findings underscore the importance of targeted interventions for FHCWs who may experience grief, guilt, and worries related to traumatic stressors. Interventions, such as trauma-informed guilt reduction therapy, may also help workers accurately assess their role in traumatic events and find positive ways to cope; however, evaluation studies are ongoing (Capone et al. 2021; Haller et al. 2020; Smith-MacDonald et al. 2018). There is also some evidence suggesting that spiritual care approaches may be helpful. For example, prior to the pandemic, one study found that higher frequency of ICU nurse interactions with chaplains was associated with decreased stress in nurses (Liberman et al. 2020).

Finally, while optimism appeared to be protective, maladaptive forms of coping, such as substance use and behavioral disengagement, were associated with an increased likelihood of having persistent distress, which is consistent with prior literature (Feder et al. 2016; Feingold et al. 2021; Løvstad et al. 2020; Mazure et al. 2000; Yoo et al. 2020). Resilience-building interventions are one strategy that may help bolster optimism and promote adaptive coping during a crisis. Computer-based resilience training delivered prior to crisis situations (Maunder et al. 2010), psychoeducational and peer support-based programs, and mental health apps may help HCWs enhance resilience to occupational and other sources of stress (DePierro et al. 2020; Golden et al. 2021), though further research is needed to evaluate their efficacy using randomized controlled designs.

There are a number of limitations of our study. The single institution design may limit the generalizability of our findings to other samples of FHCWs. Our modest response rate and sample size may limit the power and ability to find differences across groups and may raise concern for sampling bias. Nevertheless, comparison of several demographic and occupational characteristics did not reveal differences between T2 survey completers vs. non-completers. Further, comparison of gender and race/ethnicity distributions of major occupational groups in the study sample did not differ relative to those observed in Mount Sinai Hospital human resources data. Given that the survey was designed to be relatively brief, several factors could not be assessed that may contribute to persistent distress (e.g., pre-pandemic trauma history). Moreover, our administering a brief 4-item measure of pandemic-associated PTSD symptoms at T1 and the full 20 -item version at $\mathrm{T} 2$ may have under- or overestimated the prevalence of symptomatic courses of distress. To increase statistical power, we combined MDD, GAD, and PTSD screening data into a composite, transdiagnostic measure of psychological distress. However, it is likely that certain symptoms (e.g., T1 PTSD symptoms) may be differentially associated with the development of other disorders (e.g., MDD). Further research in larger samples of FHCWs is needed to evaluate this possibility, as well as to evaluate how specific symptoms of these disorders during the acute phase of the pandemic may be linked to longer-term distress and other clinical outcomes of relevance to FHCWs, such as burnout. As in any survey-based study, responses are based on perceptions and require participants to recall certain exposures and behaviors, and thus, recall bias may influence these results. Finally, the small number of FHCWs in the new-onset distress group also limited statistical power to detect differences between this group and others.

\section{Conclusions}

Results of this this longitudinal study of FHCWs following the initial surge of the COVID-19 pandemic revealed that 20.5\% had psychological distress 7 months after the surge, with pandemic-related worries, pre-pandemic burnout, lower emotional support, and feeling less valued by leaders being strongly linked to persistent distress. These results extend our previous findings (Feingold et al. 2021; Golden et al. 
2021) and highlight the need for healthcare organizations to evaluate and implement system-wide programs to reduce burnout, improve leadership support, promote a culture of psychological safety, and provide resources to support positive coping and processing of work-related traumatic events. Such efforts may help institutions move away from distress and toward growth in the wake of communal trauma.

Supplementary Information The online version contains supplementary material available at https://doi.org/10.1007/s00420-022-01832-0.

Acknowledgements The authors wish to thank all of the participants at the Mount Sinai Hospital who participated in this study.

Author contributions All the authors contributed to the study conception and design. Material preparation and data collection were performed by LP, JR, JF, and JV. Analyses were conducted by CC and RP. The first draft of the manuscript was written by LP, RP, JF, and SS, and all the authors commented on and edited previous versions of the manuscript. All the authors read and approved the final manuscript.

Funding This study was supported by internal funding devoted to COVID-19-related projects from the Icahn School of Medicine at Mount Sinai.

Availability of data and material The datasets generating during this research are not publicly available due to the confidential nature of the data with respect to mental health outcomes and their relationship to work, but may be available upon reasonable request from the corresponding author (LP).

Code availability Not applicable.

\section{Declarations}

Conflict of interest The authors report no conflicts of interests for this work.

Ethical approvals This study was approved and deemed exempt by the Institutional Review Board at the Icahn School of Medicine at Mount Sinai. HS\#: 20-00423; GCO\#1: 20-0888(0001) Icahn School of Medicine at Mount Sinai.

Standards of reporting We utilized the STROBE Checklist to adhere to reporting guidelines for observational studies.

Consent to participate As part of the exempt protocol, an informed consent document was created and approved by the Institutional Review Board at the Icahn School of Medicine at Mount Sinai, which was the first page of the survey received by participants. Participants consented by continuing on to complete the survey but no written consent was required.

Consent to publish Not applicable.

\section{References}

Alahdab F, Halvorsen AJ, Mandrekar JN, Vaa BE, Montori VM, West CP, Murad MH, Beckman TJ (2020) How do we assess resilience and grit among internal medicine residents at the Mayo Clinic? A longitudinal validity study including correlations with medical knowledge, professionalism and clinical performance. Bmj open 10:e40699

Azur MJ, Stuart EA, Frangakis C, Leaf PJ (2011) Multiple imputation by chained equations: what is it and how does it work? Int $\mathrm{J}$ Methods Psychiatr Res 20:40-49

Bohlken J, Schomig F, Lemke MR, Pumberger M, Riedel-Heller SG (2020) COVID-19 pandemic: stress experience of healthcare workers-a short current review. Psychiatr Prax 47:190-197

Cai W, Lian B, Song X, Hou T, Deng G, Li H (2020) A cross-sectional study on mental health among health care workers during the outbreak of Corona Virus Disease 2019. Asian J Psychiatr 51:102111-102211

Capone C, Norman SB, Haller M, Davis B, Shea MT, Browne K, Lang AJ, Schnurr PP, Golshan S, Afari N, Pittman J, Allard CB, Westendorf L (2021) 'Trauma Informed Guilt Reduction (TrIGR) therapy for guilt, shame, and moral injury resulting from trauma: rationale, design, and methodology of a two-site randomized controlled trial. Contemp Clin Trials 101:106251

DePierro J, Katz CL, Marin D, Feder A, Bevilacqua L, Sharma V, Hurtado A, Ripp J, Lim S, Charney D (2020) Mount Sinai's center for stress, resilience and personal growth as a model for responding to the impact of COVID-19 on health care workers. Psychiatry Res 293:113426-113526

Dong E, Du H, Gardner L (2020) An interactive web-based dashboard to track COVID-19 in real time. Lancet Inf Dis 20:533-534

Dusetzina SB, Tyree S, .Meyer AM, et al. (2014) Linking data for health services research: a framework and instructional guide, Agency for Healthcare Research and Quality, Accessed August 2. Available from: https://www.ncbi.nlm.nih.gov/books/NBK25 $3312 /$

Feder A, Mota N, Salim R, Rodriguez J, Singh R, Schaffer J, Schechter CB, Cancelmo LM, Bromet EJ, Katz CL, Reissman DB, Ozbay F, Kotov R, Crane M, Harrison DJ, Herbert R, Levin SM, Luft BJ, Moline JM, Stellman JM, Udasin IG, Landrigan PJ, Zvolensky MJ, Yehuda R, Southwick SM, Pietrzak RH (2016) Risk, coping and PTSD symptom trajectories in World Trade Center responders. J Psychiatr Res 82:68-79

Feingold JH, Peccoralo L, Chan CC, Kaplan CA, Kaye-Kauderer H, Charney D, Verity J, Hurtado A, Burka L, Syed SA, Murrough JW, Feder A, Pietrzak RH, Ripp J (2021) Psychological impact of the COVID-19 pandemic on frontline health care workers during the pandemic surge in New York City. Chronic Stress (thousand Oaks) 5:2470547020977891

Forrest, C. B., H. Xu, L. E. Thomas, L. E. Webb, L. W. Cohen, T. S. Carey, C. H. Chuang, N. M. Daraiseh, R. Kaushal, J. C. McClay, F. Modave, E. Nauman, J. V. Todd, A. Wallia, C. Bruno, A. F. Hernandez, E. C. O'Brien, and Hero Registry Research Group (2021) Impact of the early phase of the COVID-19 pandemic on US healthcare workers: results from the HERO registry. J Gen Intern Med 36:1319-1326

Galatzer-Levy IR, Huang SH, Bonanno GA (2018) Trajectories of resilience and dysfunction following potential trauma: a review and statistical evaluation. Clin Psychol Rev 63:41-55

Geier TJ, Hunt JC, Hanson JL, Heyrman K, Larsen SE, Brasel KJ, deRoon-Cassini TA (2020) Validation of Abbreviated Four- and Eight-Item Versions of the PTSD Checklist for DSM-5 in a Traumatically Injured Sample. J Trauma Stress 33:218-226

Golden EA, Zweig M, Danieletto M, Landell K, Nadkarni G, Bottinger E, Katz L, Somarriba R, Sharma V, Katz CL, Marin DB, DePierro J, Charney DS (2021) a resilience-building app to support the mental health of health care workers in the COVID-19 era: design process, distribution, and evaluation. JMIR Form Res 5:e26590

Groemping U (2006) Relative importance for linear regression in R: the package relaimpo. J Stat Softw 17:1 
Haller M, Norman SB, Davis BC, Capone C, Browne K, Allard CB (2020) A model for treating COVID-19-related guilt, shame, and moral injury. Psychol Trauma 12:S174-S176

Hennein R, Mew EJ, Lowe SR (2021) Socio-ecological predictors of mental health outcomes among healthcare workers during the COVID-19 pandemic in the United States. PLoS ONE 16:e0246602

Kisely S, Warren N, McMahon L, Dalais C, Henry I, Siskind D (2020) Occurrence, prevention, and management of the psychological effects of emerging virus outbreaks on healthcare workers: rapid review and meta-analysis. BMJ 369:m1642

Lai J, Ma S, Wang Y, Cai Z, Hu J, Wei N, Wu J, Du H, Chen T, Li R, Tan H, Kang L, Yao L, Huang M, Wang H, Wang G, Liu Z, Hu $S$ (2020) Factors associated with mental health outcomes among health care workers exposed to coronavirus disease 2019. JAMA Netw open 3:e203976

Levenshtein VI (1966) Binary codes capable of correcting deletions, insertions and reversals. Cybern Control Theory 10(8):707-710

Liberman T, Kozikowski A, Carney M, Kline M, Axelrud A, Ofer A, Rossetti M, Pekmezaris R (2020) Knowledge, attitudes, and interactions with chaplains and nursing staff outcomes: a survey study. J Relig Health 59:2308-2322

López Steinmetz LC, Herrera CR, Fong SB, Godoy JC (2021) A longitudinal study on the changes in mental health of healthcare workers during the COVID-19 Pandemic. Psychiatry 1-16. https://doi. org/10.1080/00332747.2021.1940469

Løvstad M, Månum G, Wisløff-Aase K, Hafstad GS, Ræder J, Larsen I, Stanghelle JK, Schanke AK (2020) Persons injured in the 2011 terror attacks in Norway-relationship between post-traumatic stress symptoms, emotional distress, fatigue, sleep, and pain outcomes, and medical and psychosocial factors. Disabil Rehabil 42:3126-3134

Maunder RG, Lancee WJ, Mae R, Vincent L, Peladeau N, Beduz MA, Hunter JJ, Leszcz M (2010) Computer-assisted resilience training to prepare healthcare workers for pandemic influenza: a randomized trial of the optimal dose of training. BMC Health Serv Res 10:72

Mazure CM, Bruce ML, Maciejewski PK, Jacobs SC (2000) Adverse life events and cognitive-personality characteristics in the prediction of major depression and antidepressant response. Am J Psychiatry 157:896-903

Naushad VA, Bierens JJ, Nishan KP, Firjeeth CP, Mohammad OH, Maliyakkal AM, ChaliHadan S, Schreiber MD (2019) A systematic review of the impact of disaster on the mental health of medical responders. Prehosp Disaster Med 34:632-643

O'Connor RC, Wetherall K, Cleare S, McClelland H, Melson AJ, Niedzwiedz CL, O'Carroll RE, O'Connor DB, Platt S, Scowcroft E, Watson B, Zortea T, Ferguson E, Robb KA (2020) Mental health and well-being during the COVID-19 pandemic: longitudinal analyses of adults in the UK COVID-19 Mental Health \& Wellbeing study. Br J Psychiatry 1-8

Ozer EJ, Best SR, Lipsey TL, Weiss DS (2003) Predictors of posttraumatic stress disorder and symptoms in adults: a meta-analysis. Psychol Bull 129(1):52-73

Paladino, L., R. P. Sharpe, S. C. Galwankar, F. Sholevar, C. Marchionni, T. J. Papadimos, E. Paul, B. Hansoti, M. Firstenberg, M. Garg, M. Watson, R. A. Baxter, S. P. Stawicki, and Medicine American College of Academic International (2017) Reflections on the Ebola public health emergency of international concern, Part 2: the unseen epidemic of posttraumatic stress among healthcare personnel and survivors of the 2014-2016 Ebola outbreak. J Glob Infect Dis 9:45-50

Peccoralo LA, Kaplan CA, Pietrzak RH, Charney DS, Ripp JA (2021) The impact of time spent on the electronic health record after work and of clerical work on burnout among clinical faculty. J Am Med Inform Assoc 28:938-947
Pollock A, Campbell P, Cheyne J, Cowie J, Davis B, McCallum J, McGill K, Elders A, Hagen S, McClurg D, Torrens C, Maxwell M (2020) Interventions to support the resilience and mental health of frontline health and social care professionals during and after a disease outbreak, epidemic or pandemic: a mixed methods systematic review. Cochrane Database Syst Rev 11:Cd013779

Rossi R, Socci V, Jannini TB, Pacitti F, Siracusano A, Rossi A, Di Lorenzo G (2021) Mental health outcomes among Italian health care workers during the COVID-19 pandemic. JAMA Netw open 4(11):e2136143

Sasaki N, Asaoka H, Kuroda R, Tsuno K, Imamura K, Kawakami N (2021) Sustained poor mental health among healthcare workers in COVID-19 pandemic: a longitudinal analysis of the four-wave panel survey over 8 months in Japan. J Occup Health 63:e12227

Schwartz DA, Connerney MA, Davila-Molina M, Tummalapalli SL (2021) Resident mental health at the epicenter of the COVID-19 pandemic. Acad Med 96:e16

Shanafelt TD, Gorringe G, Menaker R, Storz KA, Reeves D, Buskirk SJ, Sloan JA, Swensen SJ (2015) Impact of organizational leadership on physician burnout and satisfaction. Mayo Clin Proc 90:432-440

Shanafelt T, Ripp J, Trockel M (2020) Understanding and addressing sources of anxiety among health care professionals during the COVID-19 pandemic. JAMA 323:2133-2134

Shechter A, Diaz F, Moise N, Anstey DE, Ye S, Agarwal S, Birk JL, Brodie D, Cannone DE, Chang B, Claassen J, Cornelius T, Derby L, Dong M, Givens RC, Hochman B, Homma S, Kronish IM, Lee SAJ, Manzano W, Mayer LES, McMurry CL, Moitra V, Pham P, Rabbani L, Rivera RR, Schwartz A, Schwartz JE, Shapiro PA, Shaw K, Sullivan AM, Vose C, Wasson L, Edmondson D, Abdalla M (2020) Psychological distress, coping behaviors, and preferences for support among New York healthcare workers during the COVID-19 pandemic. Gen Hosp Psychiatry 66:1-8

Shin C, Lee SH, Han KM, Yoon HK, Han C (2019) Comparison of the usefulness of the PHQ-8 and PHQ-9 for screening for major depressive disorder: analysis of psychiatric outpatient data. Psychiatry Investig 16:300-305

Smid GE, van der Velden PG, Lensvelt-Mulders GJ, Knipscheer JW, Gersons BP, Kleber RJ (2012) Stress sensitization following a disaster: a prospective study. Psychol Med 42:1675-1686

Smid GE, Kleber RJ, Rademaker AR, van Zuiden M, Vermetten E (2013) The role of stress sensitization in progression of posttraumatic distress following deployment. Soc Psychiatry Psychiatr Epidemiol 48:1743-1754

Smith-MacDonald LA, Morin JS, Brémault-Phillips S (2018) Spiritual dimensions of moral injury: contributions of mental health chaplains in the canadian armed forces. Front Psychiatry 9:592

Spitzer RL, Kroenke K, Williams JB, Löwe B (2006) A brief measure for assessing generalized anxiety disorder: the GAD-7. Arch Intern Med 166:1092-1097

Tabakakis CK, McAllister M, Bradshaw J, To QG (2019) Psychological resilience in New Zealand registered nurses: the role of workplace characteristics. J Nurs Manag 27:1351-1358

Tonidandel S, LeBreton JM (2009) Determining the relative importance of predictors in logistic regression: an extension of relative weight analysis. Organ Res Methods 13:767-781

Wang C, Pan R, Wan X, Tan Y, Linkang Xu, McIntyre RS, Choo FN, Tran B, Ho R, Sharma VK, Ho C (2020) A longitudinal study on the mental health of general population during the COVID-19 epidemic in China. Brain Behav Immun 87:40-48

Weathers FW, Litz BT, Keane TM, Palmieri PA, Marx BP, Schnurr PP (2013) The PTSD checklist for DSM-5 (PCL-5). Accessed 6-16-21.

West CP, Dyrbye LN, Sloan JA, Shanafelt TD (2009) Single item measures of emotional exhaustion and depersonalization are useful for 
assessing burnout in medical professionals. J Gen Intern Med 24:1318-1321

West CP, Dyrbye LN, Satele DV, Sloan JA, Shanafelt TD (2012) Concurrent validity of single-item measures of emotional exhaustion and depersonalization in burnout assessment. J Gen Intern Med 27:1445-1452

Wu P, Fang Y, Guan Z, Fan B, Kong J, Yao Z, Liu X, Fuller CJ, Susser E, Lu J, Hoven CW (2009) The psychological impact of the SARS epidemic on hospital employees in China: exposure, risk perception, and altruistic acceptance of risk. Can J Psychiatry 54:302-311

Yoo S, Lee JH, Kim S (2020) Coping and rumination as mediators in the development of reactive embitterment in the general population. Psychol Trauma 13(5):565-574

Publisher's Note Springer Nature remains neutral with regard to jurisdictional claims in published maps and institutional affiliations. 